\title{
Specific Heat Study of Fulde-Ferrell-Larkin-Ovchinnikov Superconducting States in Multibands Materials Iron-Based Systems
}

\author{
D. CRivelli ${ }^{a, b}$ AND A. PtoK ${ }^{c, *}$ \\ ${ }^{a}$ Institute of Physics, University of Silesia, 40-007 Katowice, Poland \\ ${ }^{b}$ Experimentalphysik I, Universität Kassel, Heinrich-Plett-Str. 40, D-34132 Kassel, Germany \\ ${ }^{c}$ Institute of Nuclear Physics, Polish Academy of Sciences, E. Radzikowskiego 152, 31-342 Kraków, Poland \\ The Fulde-Ferrell-Larkin-Ovchinnikov phase is the superconducting state for which the Cooper pairs have \\ non-zero total momentum. From the time of conception of this phase, many groups have been searching for a \\ realization of the state. Here we describe a proposal of experimental verification of this state in the case of multi- \\ bands systems, by carrying out the specific-heat measurement.
}

DOI: 10.12693/APhysPolA.130.507

PACS/topics: $74.20 .-\mathrm{z}, 74.70 . \mathrm{Xa}$

\section{Introduction}

Superconductivity as a state with zero static electric resistance was discovered by Kamerlingh-Onnes in 1911 [1]. Bardeen, Cooper and Schrieffer (BCS) in 1957 proposed a theory allowing for a quantitative understanding of this state $[2,3]$, namely, superconductivity is made possible by the pairing of electrons and creating the spinsinglet state with zero total momentum called the Cooper pair [4]. Years later, in 1964 two groups, Fulde and Ferrell [5], Larkin and Ovchinnikov [6], proposed the phase for which the total momentum of Cooper pairs is nonzero. This state is known as the Fulde-Ferrell-LarkinOvchinnikov (FFLO) phase.

The FFLO phase can be realized in a system in which the spin splitting appears between the Fermi-surface sheets for electrons with opposite spins (cf. Fig. 1). In the BCS state, only the $(\boldsymbol{k} \uparrow)$ and $(-\boldsymbol{k} \downarrow)$ states of electrons are paired, with the total momentum equal to zero. However, when the Fermi surface is split, then electrons can be paired in the states $(\boldsymbol{k} \uparrow)$ and $(-\boldsymbol{k}+\boldsymbol{Q} \downarrow)$. This leads to the formation of FFLO state where the Cooper pairs have total momentum equal to a nonzero value $\boldsymbol{Q}$.

Since the time of the first suggestion $[5,6]$ of the FFLO state appearance, many groups researched materials in which the phase can be experimentally realized. Because the main cause of the FFLO state occurrence is the Zeeman splitting of the Fermi surface, the orbital (diamagnetic) effects should be regarded as negligible compared to the spin splitting. This relation is quantified by a relatively high value of the Maki parameter [7] $\alpha_{\mathrm{M}} \sim \sqrt{2} H_{\text {orb }} / H_{P}$. Theoretical calculations point to the lower bound $\alpha_{\mathrm{M}}>1.8$ to have a stable FFLO phase [8].

\footnotetext{
*corresponding author; e-mail: aptok@mmj.pl
}

However we must keep in mind that non-magnetic effects can also split the Fermi surface. For example, a similar effect can be achieved by the spin- or mass-imbalance in solid state $[9,10]$ or in ultra-cold fermion gases [11-13].
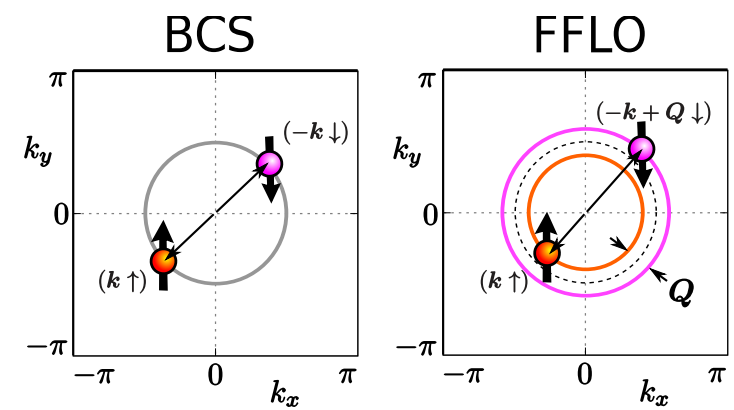

Fig. 1. Schematic presentation of the Cooper pair formation in the BCS and FFLO states. The BCS (FFLO) state occurs in the absence (presence) of spin-split Fermi-surface sheets for electrons with opposite spins. Here the Fermi surfaces for electrons with spin up and down are represented by the gray (orange and pink respectively) circle in absence (presence) of the splitting.

As already mentioned, suitable materials for the FFLO observation must have a high value of the Maki parameter $\alpha_{\mathrm{M}}$. In this situation, the critical magnetic field is determined by the Zeeman effect. Candidates for the observation of this phase are the materials close to the so-called Pauli limit, characterized by a first-order phase transition from the superconducting to normal state [1418]. Examples from this class of materials are organic superconductors [19], heavy fermion systems [20] and iron-based superconductors [21-23]. For example, in the heavy fermion $\mathrm{CeCoIn}_{5}$ compound, a possible FFLO phase has been reported by several experiments, from calorimetric measurements [24, 25], anisotropy in thermal conductivity [26], and NMR studies [27, 28]. In 
iron-based superconductors a strong anisotropy was observed in the upper critical magnetic field [21, 22], and in the Pauli limit signature [21-23]. Moreover, in our previous theoretical works we have shown that the FFLO phase is the thermodynamically-stable ground state in some iron-based superconductors within two-orbital [29] and three-orbital models [30, 31].

In both organic $[32,33]$ and heavy-fermion systems [24, 25], calorimetric measurements are the fundamental tool to probe the system under the superconducting dome. In both cases, additional peaks were found in the specific heat, which have been interpreted as signals about the phase transition from the BCS to the FFLO phase. However, the experiments require extreme conditions, since the FFLO phase exists only in the regime of high magnetic fields above the critical BCS limit, and at low temperatures near the absolute zero. In the case of multi-band iron-based superconductors, the measurements in the literature were performed at temperatures too high $[34,35]$ to account as evidence for the phase transition to the FFLO state. It is important to outline the expected calorimetric-data profile in presence of the FFLO phase transition, to facilitate the acquisition of relevant data in multi-band materials. We discuss possible results of this measurement in a paradigmatic iron-based superconductor using the three-band model proposed by Daghofer et al. (see Appendix A). Next, using standard procedures we numerically calculate the specific heat (see Appendix B). The discussion follows the computational results in Sect. 2.

\section{Numerical results and discussion}

The most general form of a momentum-dependent noninteracting Hamiltonian of a multi-orbital system in the tight-binding approximation is given by

$$
H_{0}=\sum_{\alpha \beta} \sum_{\boldsymbol{k} \sigma}\left(T_{\boldsymbol{k}}^{\alpha \beta}-(\mu+\sigma \mu) \delta_{\alpha \beta}\right) c_{\alpha \boldsymbol{k} \sigma}^{\dagger} c_{\beta \boldsymbol{k} \sigma},
$$

using $c_{\alpha \boldsymbol{k} \sigma}\left(c_{\alpha \boldsymbol{k} \sigma}^{\dagger}\right)$ to denote the annihilation (creation) operator of electrons with spin $\sigma$ and momentum $\boldsymbol{k}$ in the orbital $\alpha . T_{k}^{\alpha \beta}$ are hopping terms between orbitals $\alpha$ and $\beta$ with momentum $\boldsymbol{k}$, determined by the model, which in the present case is the three orbital model of iron-based superconductors described in Appendix A. Band structures are reconstructed by diagonalizing $H_{0}=\sum_{\varepsilon \boldsymbol{k} \sigma} E_{\varepsilon \boldsymbol{k} \sigma} d_{\varepsilon \boldsymbol{k} \sigma}^{\dagger} d_{\varepsilon \boldsymbol{k} \sigma}$, in which now the operators $d_{\alpha \boldsymbol{k} \sigma}$ correspond to annihilation of electrons in band $\varepsilon[36]$.

In the language of band-basis operators, the interaction part of the Hamiltonian can be written in the phenomenological form

$$
H_{S C}=\sum_{\varepsilon \boldsymbol{k}}\left(\Delta_{\varepsilon \boldsymbol{k}} d_{\varepsilon \boldsymbol{k} \uparrow}^{\dagger} d_{\varepsilon,-\boldsymbol{k}+\boldsymbol{Q}_{\varepsilon \downarrow}}^{\dagger}+H . c .\right),
$$

where $\Delta_{\varepsilon \boldsymbol{k}}=\Delta_{\varepsilon} \eta(\boldsymbol{k})$ is the superconducting order parameter in band $\varepsilon$ with amplitude $\Delta_{\varepsilon}$ and symmetry $\eta(\boldsymbol{k})$ (e.g. equal to unity for $s$-wave [30]), while $\boldsymbol{Q}_{\varepsilon}$ is the total momentum of the Cooper pairs in the band.
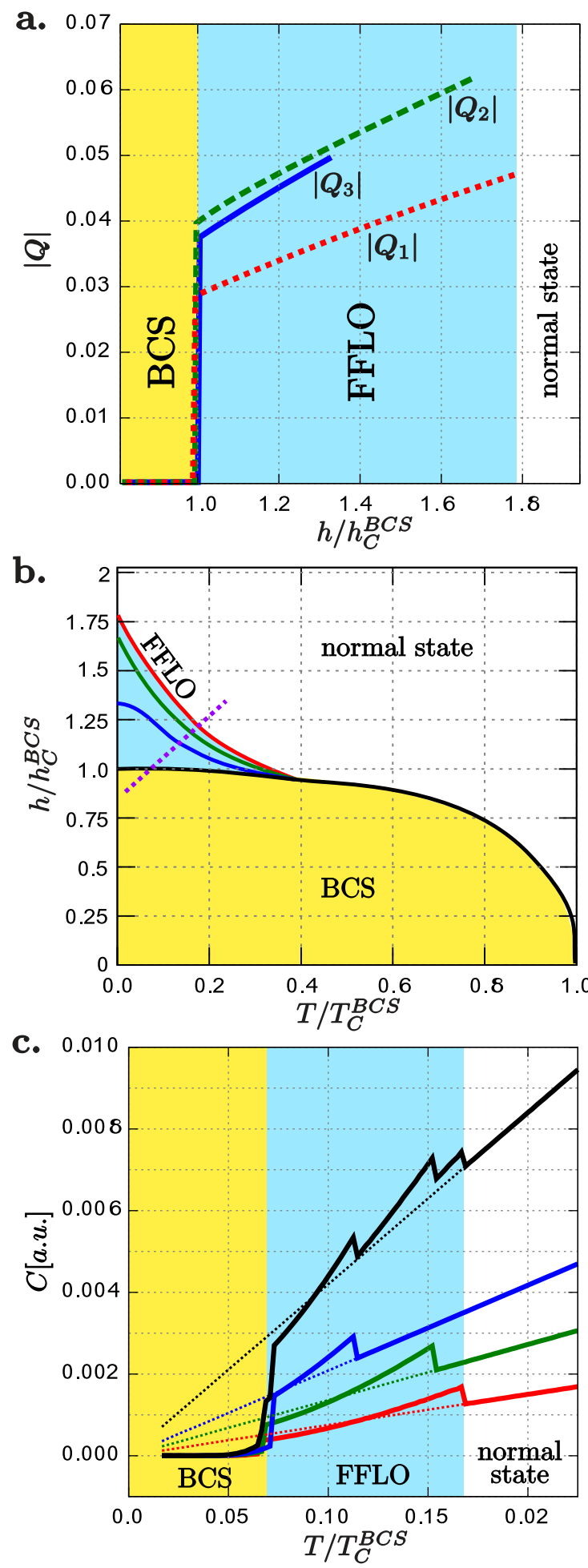

Fig. 2. (a) Change of the Cooper pair total momentum in each band. (b) Temperature vs. magnetic-field phase diagram. (c) Specific heat $C$ in each band along the temperatures and magnetic fields marked by the cyan dotted line in part (b), where the black line denotes the total heat capacity summed over the three bands. The line colors red, green and blue correspond to bands 1 , 2 , and 3 , respectively. 
The full set of interactions for the system is given by the total Hamiltonian $H=H_{0}+H_{S C}$. The grand canonical potential can be calculated from its definition $\left.\Omega \equiv k_{B} T \ln 9 \operatorname{Tr}\left(\exp \left(-H / k_{B} T\right)\right)\right)$, while the thermodynamically stable state is found through the minimization of $\Omega$ with respect to the amplitude of the superconducting order parameter $\Delta_{\varepsilon}$ and the total momentum of the Cooper pairs $\boldsymbol{Q}_{\varepsilon}$, keeping the magnetic field $h$ and temperature $T$ fixed. Additionally, we calculated the entropy and specific heat using the procedure described in Appendix B.

For simplicity, we present results for $\eta(\boldsymbol{k})=1$, corresponding to a $s$-wave symmetry of the superconducting order parameter. From the minimization of $\Omega$ we can numerically find $\Delta_{\varepsilon}$ and $\boldsymbol{Q}_{\varepsilon}$ in each band $\varepsilon$. To calculate $\Omega$, the procedure described in Ref. [31] is employed, for a square lattice of size $N_{x} \times N_{y}=10000 \times 10000$, which makes finite-size effects negligible [37].

The total momentum of the Cooper pairs for $T \rightarrow 0$ is shown in Fig. 2a. In every band the BCS type superconductivity vanishes at the same critical magnetic field $h_{C}^{B C S}$. At sufficiently low temperatures above this field, the FFLO phase can be found. However, the FFLO superconductivity in each band vanishes at a different critical magnetic field $\left(h_{C}^{F F L O}\right)_{\varepsilon}$. Moreover, at a constant value of the magnetic field, the shape and splitting of the Fermi-surface sheets is different and band dependent, leading to differing values of the total Cooper pair momentum at every non-zero temperature. Further consequences of the difference between bands are observed in Fig. 2b, in which we present the $h-T$ (magnetic fieldtemperature) phase diagram. Raising the temperature of the system in the FFLO state, many phase transitions appear, each caused by the successive disappearance of the FFLO phase in each band. Consequently, this set of transitions could be experimentally observed using calorimetry measurements. In our case, calculating numerically the specific heat in each band by differentiation of Eq. (B1), we find peaks corresponding to each of the phase transitions. Examples of this are shown in Fig. 2c, where we have calculated the specific heat along a path in the $h-T$ diagram.

\section{Summary}

In conclusion, in this paper we stated the main conditions needed for the realization of the Fulde-FerrellLarkin-Ovchinnikov phase. We also present specific heat calculations for multi-band materials, to be used as a basis for experimental verification in iron-based superconductors. Additional peaks in the specific heat carry information about the FFLO phase [38]. A big advantage of the presented methods is the applicability to systems sensitive to finite size effects [39, 40].

\section{Acknowledgments}

The authors are thankful to Konrad J. Kapcia, Przemysław Piekarz and Andrzej Ślebarski for very fruitful discussions and comments. This work was supported by the Ministry of Education, Youth, and Sports from the Large Infrastructures for Research, Experimental Development, and Innovations project IT4 Innovations National Supercomputing Center - LM2015070.

\section{Appendix A: Three orbital model of iron-based superconductors}

We describe iron-based superconductors using the three-band model proposed by Daghofer et al. in Refs. [41, 42]. This model selects the $d_{x z}, d_{y z}$, and $d_{x y}$ orbitals of iron (labeled as $\alpha=1,2,3$, respectively). Hopping terms of the model are given by

$$
\begin{aligned}
& T_{\boldsymbol{k}}^{11}=2 t_{2} \cos k_{x}+2 t_{1} \cos k_{y}+4 t_{3} \cos k_{x} \cos k_{y} \\
& \quad+2 t_{11}\left(\cos \left(2 k_{x}\right)-\cos \left(2 k_{y}\right)\right) \\
& \quad+4 t_{12} \cos \left(2 k_{x}\right) \cos \left(2 k_{y}\right), \\
& T_{\boldsymbol{k}}^{22}=2 t_{1} \cos k_{x}+2 t_{2} \cos k_{y}+4 t_{3} \cos k_{x} \cos k_{y} \\
& \quad-2 t_{11}\left(\cos \left(2 k_{x}\right)-\cos \left(2 k_{y}\right)\right) \\
& \quad+4 t_{12} \cos \left(2 k_{x}\right) \cos \left(2 k_{y}\right), \\
& T_{\boldsymbol{k}}^{33}=\epsilon_{0}+2 t_{5}\left(\cos k_{x}+\cos k_{y}\right) \\
& \quad+4 t_{6} \cos k_{x} \cos k_{y}+2 t_{9}\left(\cos \left(2 k_{x}\right)-\cos \left(2 k_{y}\right)\right) \\
& \quad+4 t_{10}\left(\cos \left(2 k_{x}\right) \cos k_{y}+\cos k_{x} \cos \left(2 k_{y}\right)\right), \\
& T_{\boldsymbol{k}}^{12}=T_{\boldsymbol{k}}^{21}=4 t_{4} \sin k_{x} \sin k_{y}, \\
& T_{\boldsymbol{k}}^{13}=\bar{T}_{\boldsymbol{k}}^{31}=2 \mathrm{i} t_{7} \sin k_{x}+4 \mathrm{i} t_{8} \sin k_{x} \cos k_{y}, \\
& T_{\boldsymbol{k}}^{23}=\bar{T}_{\boldsymbol{k}}^{32}=2 \mathrm{i} t_{7} \sin k_{y}+4 \mathrm{i} t_{8} \sin k_{y} \cos k_{x} .
\end{aligned}
$$

In Ref. [42], the parameters in electron-volts are given as: $t_{1}=-0.08, t_{2}=0.1825, t_{3}=0.08375, t_{4}=-0.03$, $t_{5}=0.15, t_{6}=0.15, t_{7}=-0.12, t_{8}=0.06, t_{9}=0.0$, $t_{10}=-0.024, t_{11}=-0.01, t_{12}=0.0275$ and $\epsilon_{0}=0.75$. The average number of particles $n=4$ is obtained by setting the chemical potential to $\mu=0.4748$ [36].

\section{Appendix B: Specific heat calculation}

Keeping the parameters $h$ and $T$ fixed, the grandcanonical potential can be treated as a function of the amplitude of the superconducting order parameter $\Delta_{\varepsilon}$ and total momentum of the Cooper pairs $\boldsymbol{Q}_{\varepsilon}$. Then $\Omega=\Omega\left(\Delta_{\varepsilon}, \boldsymbol{Q}_{\varepsilon}\right)$, while the entropy $S=-\mathrm{d} \Omega / \mathrm{d} T$ and superconducting specific heat at temperature $T$ is $C=$ $-T \partial^{2} \Omega / \partial T^{2}[43-45]$. Here

$$
\begin{aligned}
& \frac{\mathrm{d} \Omega}{\mathrm{d} T}= \\
& \sum_{\varepsilon}\left[\left(\frac{\partial \Omega_{\varepsilon}}{\partial T}\right)_{e}+\left(\frac{\partial \Omega_{\varepsilon}}{\partial \Delta_{\varepsilon}}\right)_{e} \frac{\partial \Delta_{\varepsilon}}{\partial T}+\left(\frac{\partial \Omega_{\varepsilon}}{\partial \boldsymbol{Q}_{\varepsilon}}\right)_{e} \frac{\partial \boldsymbol{Q}_{\varepsilon}}{\partial T}\right],
\end{aligned}
$$

where the subscript e labels the equilibrium values of $\Delta_{\varepsilon}$ and $\boldsymbol{Q}_{\varepsilon}$, and the sum is over the bands $\varepsilon$. 
From the thermodynamical-equilibrium condition, we have $\left(\partial \Omega_{\varepsilon} / \partial \Delta_{\varepsilon}\right)_{e}=\left(\partial \Omega_{\varepsilon} / \partial \boldsymbol{Q}_{\varepsilon}\right)_{e}=0$ in all bands $\varepsilon$, since $\Omega\left(\Delta_{\varepsilon}, \boldsymbol{Q}_{\varepsilon}\right)$ is at a minimum. Hence $S=$ $-\sum_{\varepsilon}\left(\partial \Omega_{\varepsilon} / \partial T\right)_{e}$. The specific heat is then defined in the usual manner by $C=-T \sum_{\varepsilon}\left(\partial^{2} \Omega_{\varepsilon} / \partial T^{2}\right)_{e}$.

\section{References}

[1] H. Kamerlingh-Onnes, Commun. Phys. Lab. Univ. Leiden 12, 120 (1911).

[2] J. Bardeen, L.N. Cooper, J.R. Schrieffer, Phys. Rev. 106, 162 (1957).

[3] J. Bardeen, L.N. Cooper, J.R. Schrieffer, Phys. Rev. 108, 1175 (1957).

[4] L.N. Cooper, Phys. Rev. 104, 1189 (1956).

[5] P. Fulde, R.A. Ferrell, Phys. Rev. 135, A550 (1964).

[6] A.I. Larkin, Yu.N. Ovchinnikov, Zh. Eksp. Teor. Fiz. 47, 1136 (1964) [Sov. Phys. JETP 20, 762 (1965)].

[7] K. Maki, T. Tsuneto, Prog. Theor. Phys. 31, 945 (1964).

[8] L.W. Gruenberg, L. Gunther, Phys. Rev. Lett. 16 , 996 (1966).

[9] J. Kaczmarczyk, J. Spałek, Phys. Rev. B 79, 214519 (2009).

[10] M.M. Maśka, M. Mierzejewski, J. Kaczmarczyk, J. Spałek, Phys. Rev. B 82, 054509 (2010).

[11] R. Mendoza, M. Fortes, M.A. Solís, Z. Koinov, Phys. Rev. A 88, 033606 (2013).

[12] S. Pahl, Z. Koinov, J. Low Temp. Phys. 176, 113 (2014).

[13] D.E. Sheehy, Phys. Rev. A 92, 053631 (2015).

[14] M. Mierzejewski, M.M. Maśka, Phys. Rev. B 69, 054502 (2004).

[15] K. Kapcia, S. Robaszkiewicz, R. Micnas, J. Phys. Condens. Matter 24, 215601 (2012).

[16] K. Kapcia, S. Robaszkiewicz, J. Phys. Condens. Matter 25, 065603 (2013).

[17] K. Kapcia, J. Supercond. Nov. Magn. 27, 913 (2014).

[18] K.J. Kapcia, Acta Phys. Pol. A 126, A-53 (2014).

[19] R. Beyer, J. Wosnitza, Low Temp. Phys. 39, 225 (2013).

[20] Y. Matsuda, H. Shimahara, J. Phys. Soc. Jpn. 76 051005 (2007).

[21] S. Khim, B. Lee, J.W. Kim, E.S. Choi, G.R. Stewart, K.H. Kim, Phys. Rev. B 84, 104502 (2011).

[22] K. Cho, H. Kim, M.A. Tanatar, Y.J. Song, Y.S. Kwon, W.A. Coniglio, C.C. Agosta, A. Gurevich, R. Prozorov, Phys. Rev. B 83, 060502 (2011).

[23] D.A. Zocco, K. Grube, F. Eilers, T. Wolf, H. v. Löhneysen, Phys. Rev. Lett. 111, 057007 (2013).
[24] A. Bianchi, R. Movshovich, N. Oeschler, P. Gegenwart, F. Steglich, J.D. Thompson, P.G. Pagliuso, J.L. Sarrao, Phys. Rev. Lett. 89, 137002 (2002).

[25] A. Bianchi, R. Movshovich, C. Capan, P.G. Pagliuso, J.L. Sarrao, Phys. Rev. Lett. 91, 187004 (2003).

[26] C. Capan, A. Bianchi, R. Movshovich, A.D. Christianson, A. Malinowski, M.F. Hundley, A. Lacerda P.G. Pagliuso, J.L. Sarrao, Phys. Rev. B 70, 134513 (2004).

[27] K. Kumagai, M. Saitoh, T. Oyaizu, Y. Furukawa, S. Takashima, M. Nohara, H. Takagi, Y. Matsuda, Phys. Rev. Lett. 97, 227002 (2006).

[28] V.F. Mitrović, M. Horvatić, C. Berthier, G. Knebel, G. Lapertot, J. Flouquet, Phys. Rev. Lett. $\mathbf{9 7}$ 117002 (2006).

[29] A. Ptok, D. Crivelli, J. Low Temp. Phys. 172, 226 (2013).

[30] D. Crivelli, A. Ptok, Acta Phys. Pol. A 126, A-16 (2014).

[31] M. Januszewski, A. Ptok, D. Crivelli, B. Gardas, Comput. Phys. Commun. 192, 220 (2015).

[32] R. Lortz, Y. Wang, A. Demuer, P.H.M. Böttger, B. Bergk, G. Zwicknagl, Y. Nakazawa, J. Wosnitza, Phys. Rev. Lett. 99, 187002 (2007).

[33] R. Beyer, B. Bergk, S. Yasin, J.A. Schlueter, J. Wosnitza, Phys. Rev. Lett. 109, 027003 (2012).

[34] J.S. Kim, E.G. Kim, G.R. Stewart, X.H. Chen, X.F. Wang, Phys. Rev. B 83, 172502 (2011).

[35] M. Abdel-Hafiez, S. Aswartham, S. Wurmehl, V. Grinenko, C. Hess, S.L. Drechsler, S. Johnston, A.U. Wolter, B. Büchner, H. Rosner, L. Boeri, Phys. Rev. B 85, 134533 (2012).

[36] A. Ptok, D. Crivelli, K.J. Kapcia, Supercond. Sci Technol. 28, 045010 (2015).

[37] A. Ptok, D. Crivelli, Comm Comp. Phys., accepted.

[38] A. Ptok, J. Phys. Condens. Matter 27, 482001 (2015).

[39] K. Aoyama, R. Beaird, D.E. Sheehy, I. Vekhter, Phys. Rev. Lett. 110, 177004 (2013).

[40] P. Wójcik, M. Zegrodnik, J. Spałek, Phys. Rev. B 91, 224511 (2015)

[41] M. Daghofer, A. Nicholson, A. Moreo, E. Dagotto, Phys. Rev. B 81, 014511 (2010).

[42] M. Daghofer, A. Nicholson, A. Moreo, Phys. Rev. B 85, 184515 (2012)

[43] M.M. Wysokiński, J. Spałek, J. Phys. Condens. Matter 26, 055601 (2014).

[44] K.J. Kapcia, S. Murawski, W. Kłobus, S. Robaszkiewicz, Physica A 437, 218 (2015).

[45] K.J. Kapcia, W. Kłobus, S. Robaszkiewicz, Acta Phys. Pol. A 127, 284 (2015). 